\title{
Rare Kaon Decays at CERN SPS
}

\author{
Venelin Kozhuharov ${ }^{1}$ \\ Atomic Phys. Dept., Faculty of Physics, University of Sofia, 5 J. Bourchier Str., 1164 Sofia, Bulgaria
}

\begin{abstract}
.
Since their discovery the K-mesons continue to provide valuable information to the theory of the interactions between the elementary particles. In two years of data taking the NA48/2 experiment at CERN SPS recorded more than 4 billion $K^{ \pm} \rightarrow \pi^{+} \pi^{-} \pi^{ \pm}$decays devoted to the search of CP violation in the charged kaon system. The huge statistics allows also a precise study of rare kaon decays. The results from the investigation of $K^{ \pm} \rightarrow \pi^{ \pm} e^{+} e^{-}$decay will be shown with their interpretation within several theoretical models. The continuation of the kaon program at CERN is the NA62 experiment devoted to the measurement of $\operatorname{Br}\left(K^{ \pm} \rightarrow \pi^{ \pm} v \bar{v}\right)$ decay with a precision of $10 \%$ in two years of data taking. The planned detector setup and the expected background and signal events will be presented. During 2007 a dedicated run devoted to NA62 prototype tests and study of $K_{e 2}$ decays was taken. The preliminary results from the study of $\operatorname{Ke} 2$ decays and the ratio $R_{K}=\Gamma\left(K^{ \pm} \rightarrow e^{ \pm} v\right) / \Gamma\left(K^{ \pm} \rightarrow \mu^{ \pm} v\right)$ at the NA48/2 and NA62 experiments will be shown.
\end{abstract}

Keywords: kaon decays, rare decays, standard model, new physics

PACS: $13.20 . \mathrm{Eb}, 14.40 . \mathrm{Aq}, 12.15 . \mathrm{Hh}, 12.60 . \mathrm{Jv}$

\section{INTRODUCTION}

Kaons contain a strange quark in their structure and because of that they decay via weak interactions. Since the initial state is made of quarks a low energy effective theory of the strong interactions is necessary for its description. The probability for the rare processes is low because they are suppressed within the Standard Model by some mechanism. Some of them proceed as flavour changing neutral currents while others have low rates due to helicity suppression. They are interesting because rates for some of them are easily calculable by means of only fundamental parameters. Since they are rare the contribution of possible New Physics can be non negligible.

\section{NA48/2 EXPERIMENTAL SETUP}

The NA48/2 experiment was primarily designed to measure the charge asymmetry in the $K^{ \pm} \rightarrow 3 \pi$ decays in order to probe for $\mathrm{CP}$ violation in the charged kaon system [1]. Kaons at the NA48/2 experiment are produced by a 400 $\mathrm{GeV} / \mathrm{c}$ primary proton beam from the CERN SPS hitting a beryllium target. A system of collimators and achromats selects particles with mean momentum of $60 \mathrm{GeV} / \mathrm{c}$ with both positive and negative charge. The momentum of the particles is measured by a beam spectrometer. The simultaneous $K^{ \pm}$beams enter a $114 \mathrm{~m}$ long evacuated decay region closed by a Kevlar window. The decays products are detected by the NA48 detector complex including:

- Magnetic spectrometer (DCH): Composed by four drift chambers and a dipole magnet deflecting the charged particles in the horizontal plane. The resulting resolution on the momentum measurement is $\delta p / p=1 \% \oplus 0.044 \% \times p$ $[\mathrm{GeV} / \mathrm{c}]$.

- Charged hodoscope (HOD): Fast scintillator detector with time resolution of $150 \mathrm{ps}$ used in the trigger system.

- Electromagnetic Calorimeter (LKR): A Liquid Krypton calorimeter of 27 radiation lengths used to measure the energy of the electrons and photons with resolution of $\delta E / E=3.2 \% / \sqrt{E} \oplus 9 \% / E \oplus 0.42 \%[\mathrm{GeV}]$.

The mean beam axis is close to the $Z$ axis of the experiment. A more detailed description of the experimental setup can be found elsewhere [2]

To study the response of the detector system a GEANT3 [3] based simulation is used. It includes full material description, local inefficiencies and time variation of the beam conditions.

\footnotetext{
${ }^{1}$ for the NA48/2 and NA62 collaborations
} 
Data were taken in 2003 and 2004 providing approximately $4 \times 10^{9} K^{ \pm} \rightarrow \pi^{ \pm} \pi^{+} \pi^{-}$decays. Apart from the nominal running conditions the data taking also included two special runs with reduced beam intensity and special trigger setup devoted to the investigation of kaon semileptonic decays. The huge statistics collected allowed to study also rare kaon decays.

\section{STUDY OF THE DECAY $K^{ \pm} \rightarrow \pi^{ \pm} e^{+} e^{-}$}

The decays $K^{ \pm} \rightarrow \pi^{ \pm} e^{+} e^{-}$are flavour changing neutral current processes and proceed through a virtual photon exchange $K \rightarrow \pi \gamma^{*}$ with a consequent transition $\gamma^{*} \rightarrow l^{+} l^{-}$. The matrix element is dominated by long distance contribution which is described within the Chiral Perturbation Theory (ChPT). The differential width can be written as a function of one kinematics variable $z=M_{l l}^{2} / M_{K}^{2}[4]$

$$
\frac{d \Gamma}{d z}=\rho(z) *|W(z)|^{2}
$$

where $\rho(z)$ is phase spase factor and $W(z)$ contains the non-trivial part of the matrix element. Several theoretical models have been proposed for the description of $W(z)$

- Model 1 - polynomial: $W(z)=G_{F} M_{K}^{2} f_{0}(1+\delta z)$ with two free parameters - $f_{0}$ and $\delta$. $G_{F}$ is the Fermi constant and $M_{K}$ is the kaon mass.

- Model 2 - ChPT at $O\left(p^{6}\right): W(z)=G_{F} M_{K}^{2}\left(a_{+}+b_{+} z\right)+W^{\pi \pi}(z)$, where the free parameters are $a_{+}$and $b_{+}$and the complete form of the pion loop term $W^{\pi \pi}(z)$ can be found in [4].

- Model 3 - ChPT together with Large- $N_{c}$ QCD: $W(z)=W(w, \beta, z)[5]$.

- Model 4 - mesonic ChPT: $W(z)=W\left(M_{a}, M_{\rho}, z\right)$ where $M_{a}$ and $M_{\rho}$ are the resonance masses [6].

The knowledge of the form-factor dependence on $z$ is important in order to obtain the total branching fraction for this decay. The reason is that the accessible kinematical region is experimentally limited due to the contribution of $K^{ \pm} \rightarrow \pi^{ \pm} \pi^{0} \rightarrow \pi^{ \pm} e^{+} e^{-} \gamma$ for $z$ below 0.08 .

In the analysis of $K^{ \pm} \rightarrow \pi^{ \pm} e^{+} e^{-}$decay the data taken during the nominal conditions in 2003 and 2004 was used. The decay $K^{ \pm} \rightarrow \pi^{ \pm} \pi^{0} \rightarrow \pi^{ \pm} e^{+} e^{-} \gamma$ was exploited as a normalization channel. The final states of both modes contain the same charged particles leading to common selection criteria and cancellation of most of the systematics.

The events were required to have a vertex from three tracks with total charge \pm 1 reconstructed in the fiducial decay region of the experiment. The three tracks had to be within the geometrical acceptance of DCH, HOD and LKR. They also had to be enough separated at the first drift chamber in order to suppress background from photon conversions and at the LKR - to avoid cluster overlapping. Particle identification was based on the ratio $E / p c$ where $p$ is the track momentum measured from the DCH and $E$ is the reconstructed energy of the particle in the LKR. Since the electrons leave all their energy in the electromagnetic calorimeter this variable peaks at one for them and for pions it was required to be $<0.85$. In order to have good energy and momentum measurement all three tracks were required to have momentum higher than $5 \mathrm{GeV} / \mathrm{c}$.

The sample of $K^{ \pm} \rightarrow \pi^{ \pm} e^{+} e^{-}$events was selected by additional requirements on the kinematics of the event. The dominant background, namely $K^{ \pm} \rightarrow \pi^{ \pm} \pi^{0} \rightarrow \pi^{ \pm} e^{+} e^{-} \gamma$, was suppressed by the condition $z>0.08$, corresponding to $M_{e e}>140 \mathrm{MeV} / \mathrm{c}^{2}$. Also a cut on the reconstructed total and transverse momentum of the event was imposed. In order to obtain the $K^{ \pm} \rightarrow \pi^{ \pm} \pi^{0} \rightarrow \pi^{ \pm} e^{+} e^{-} \gamma$ sample additional cluster in the LKR with no associated track was required. The energy of the cluster together with the $e^{+} e^{-}$pair had to compatible with $\pi^{0} \rightarrow e^{+} e^{-} \gamma$ decay.

With the presented selection $7253 K^{ \pm} \rightarrow \pi^{ \pm} e^{+} e^{-}$were reconstructed. Their invariant mass distribution is shown on Figure 1(a) where the agreement with the simulation is also visible. The background contribution is $(1 \pm 0.1) \%$ measured from data. The number of $K^{ \pm} \rightarrow \pi^{ \pm} \pi^{0} \rightarrow \pi^{ \pm} e^{+} e^{-} \gamma$ events was $1.212 \times 10^{7}$.

In order to obtain the ratio of the branching fractions these two number of events had to be corrected for the different trigger efficiency and acceptance for which Monte Carlo was used. This allowed to obtain a model independent value for the branching fraction of $K^{ \pm} \rightarrow \pi^{ \pm} e^{+} e^{-}$only in the kinematically accessible region

$$
B r\left(K^{ \pm} \rightarrow \pi^{ \pm} e^{+} e^{-}\right)_{M_{e e}<140 \mathrm{MeV} / c^{2}}=\left(2.28 \pm 0.03_{\text {stat }} \pm 0.04_{\text {syst }} \pm 0.06_{\text {ext }}\right) \times 10^{-7}
$$

where the dominant systematic uncertainty comes from the particle identification and simulation of the beam and the radiative corrections. The external error is due to the knowledge of $\operatorname{Br}\left(K^{ \pm} \rightarrow \pi^{ \pm} \pi^{0} \rightarrow \pi^{ \pm} e^{+} e^{-} \gamma\right)$. 
(a)

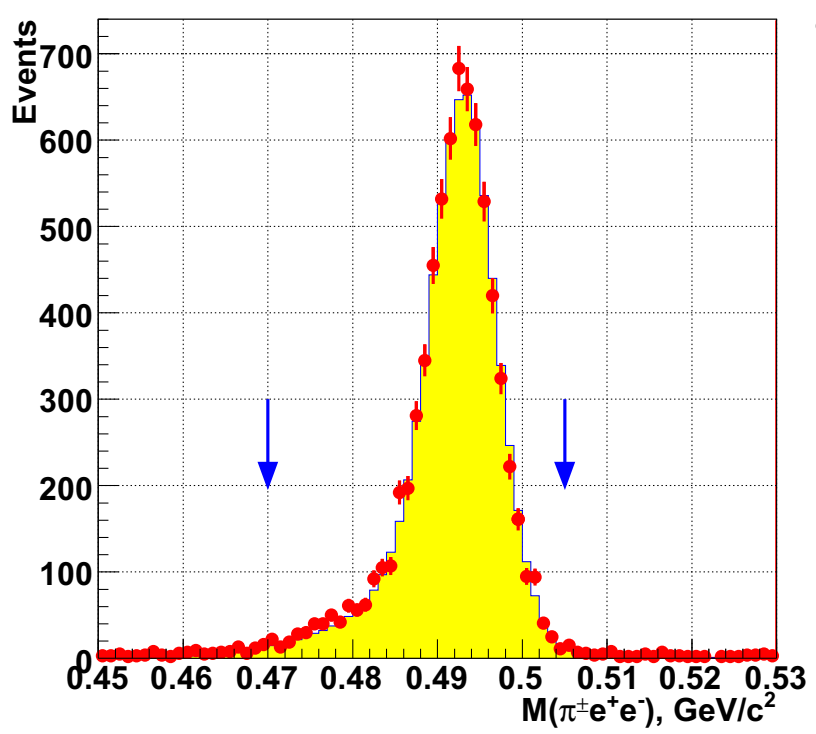

(b)

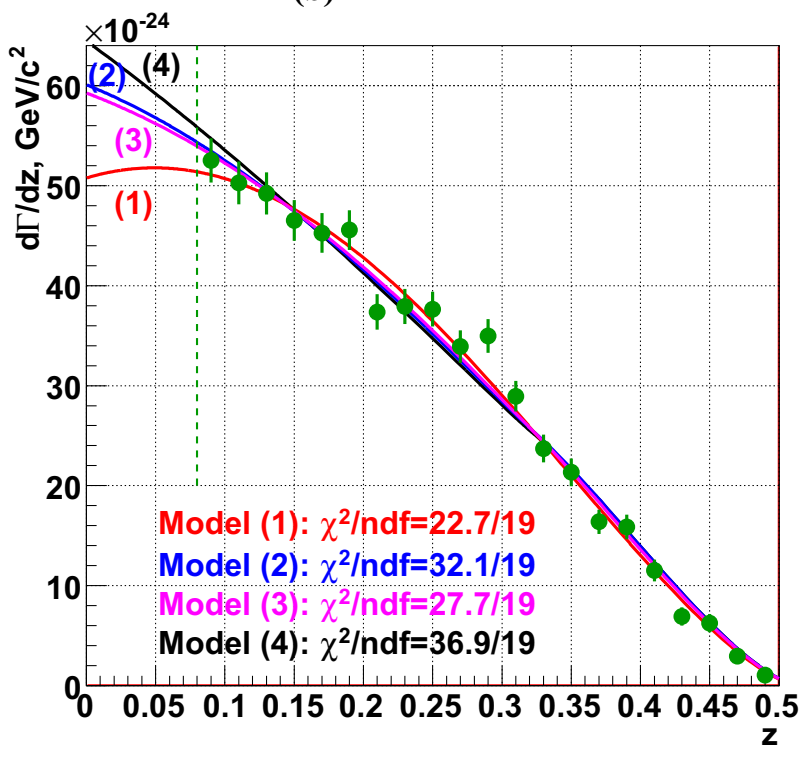

FIGURE 1. Invariant mass distribution (a) for the reconstructed data events (dots) and Monte Carlo (histogram) and fits to the data using four different theoretical models for the matrix element (b).

The total branching fraction for the decay $K^{ \pm} \rightarrow \pi^{ \pm} e^{+} e^{-}$was obtained by extrapolation of the experimental $z$ spectrum to the whole $z$ kinematics region. This was done by using the four presented parametrization of the matrix element. For each of them the corresponding free parameters were obtained by fit to the data and the resulting distributions are presented on Figure 1(b). As can be seen all four models describe the data well. For each of them a separate value for the branching fraction was calculated. In order to obtain a model independent result the combined value was used with attributed error due to the maximal difference of the model dependent values, leading to

$$
\operatorname{Br}\left(K^{ \pm} \rightarrow \pi^{ \pm} e^{+} e^{-}\right)=\left(3.11 \pm 0.04_{\text {stat }} \pm 0.05_{\text {syst }} \pm 0.08_{\text {ext }} \pm 0.07_{\text {model }}\right) \times 10^{-7}
$$

which is in agreement with the previous experimental measurements [7]. Figure 1(b) shows also that the decay $K^{ \pm} \rightarrow \pi^{ \pm} e^{+} e^{-}$is described well within the ChPT.

$$
\text { MEASUREMENT OF } R_{K}=\Gamma\left(K^{ \pm} \rightarrow e^{ \pm} v\right) / \Gamma\left(K^{ \pm} \rightarrow \mu^{ \pm} v\right)
$$

In the Standard Model (SM) the weak interactions of the leptons are described by a unique coupling constant $G_{F}$. This universality can be tested by investigating the leptonic decays of light mesons like $K^{ \pm} \rightarrow l^{ \pm} v(K l 2)$ decays. They proceed as tree level processes within the SM and the ratio between of the decay widths of the electronic and muonic mode $R_{K}=\Gamma(K e 2) / \Gamma(K \mu 2)$ can be written as [8]

$$
R_{K}=\frac{m_{e}^{2}}{m_{\mu}^{2}}\left(\frac{m_{K}^{2}-m_{e}^{2}}{m_{K}^{2}-m_{\mu}^{2}}\right)\left(1+\delta R_{K}\right)
$$

where the term $\delta R_{K}=-(3.78 \pm 0.04) \%$ represents the radiative corrections. In the ratio $R_{K}$ the theoretical uncertainties on the hadronic matrix element cancel resulting in a precise prediction $R_{K}=(2.477 \pm 0.001) \times 10^{-5}[9]$.

Considering different extensions of the SM (like models with Lepton Flavour Violation, different SUSY scenarios) a constructive or destructive contribution to $R_{K}$ as high as $3 \%$ could be achieved [10].

Two preliminary measurement of $R_{K}$ were performed within NA48/2 collaboration [11] which allowed to optimize the analysis strategy. The following measurement is obtained within the NA62 experiment which profitted from the NA48 beam line and detector setup. Kaon beam with mean momentum of $74 \mathrm{GeV}$ was used. $90 \%$ of the data was taken with only $K^{+}$beam and approximately $10 \%$ with simultaneous $K^{+}$and $K^{-}$beams. 
The similarity between the two decays allows to exploit systematics cancellations by using common selection criteria. The events were required to have only one reconstructed charged track within the DCH, LKR and HOD geometrical acceptance with momentum in the interval $15 \mathrm{GeV} / c<p<65 \mathrm{GeV} / \mathrm{c}$. In order to suppress the background a cut on extra non-associated with the track LKR clusters with energy above $2 \mathrm{GeV}$ was made. The track had to originate from a vertex within a defined decay volume. The particle identification was based on the $E / p c$ variable which had to be in the interval $0.95<E / p c<1.1$ for electrons and less than 0.85 for muons. Under the assumption of the particle type the missing mass squared was calculated $M_{\text {miss }}^{2}=\left(P_{K}-P_{l}\right)^{2}$ where $P_{K}\left(P_{l}\right)$ is the kaon (lepton) four momentum. A momentum dependent cut of $\left|M_{\text {miss }}^{2}\right|<M_{0}^{2}$ was used with $M_{0}^{2}$ varying in the interval 0.009 to 0.013 $\left(\mathrm{GeV} / \mathrm{c}^{2}\right)^{2}$.

The dominant background contribution in the $K_{e 2}$ sample was identified to come from $K_{\mu 2}$ events with muons leaving all their energy in the electromagnetic calorimeter. The missing mass squared under electron hypothesis for both $K_{e 2}$ and $K_{\mu 2}$ events is shown on Figure 2(a). The two decays are well separated below $35 \mathrm{GeV} / \mathrm{c}$ track momentum but completely overlap kinematically for higher values. In order to estimate the amount of muons with $0.95<E / p<1.1$ a special Geant4 based Monte Carlo simulation was employed, which was validated by events from a special muon run taken with a $10 X_{0}$ thick $\mathrm{Pb}$ wall placed in front of the LKR. A systematic uncertainty corresponding to the statistics of the validation sample was attributed to the background estimation. The missing mass distribution for the reconstructed $K_{e 2}$ data events together with the simulation of the signal and backgrounds are shown on Figure 2(b). The background was estimated to be $(8.03 \pm 0.23) \%$ of the $K_{e 2}$ sample. A total of $\sim 51000 K_{e 2}$ and $15.56 \times 10^{6}$ $K_{\mu 2}$ candidates were reconstructed.

(a)
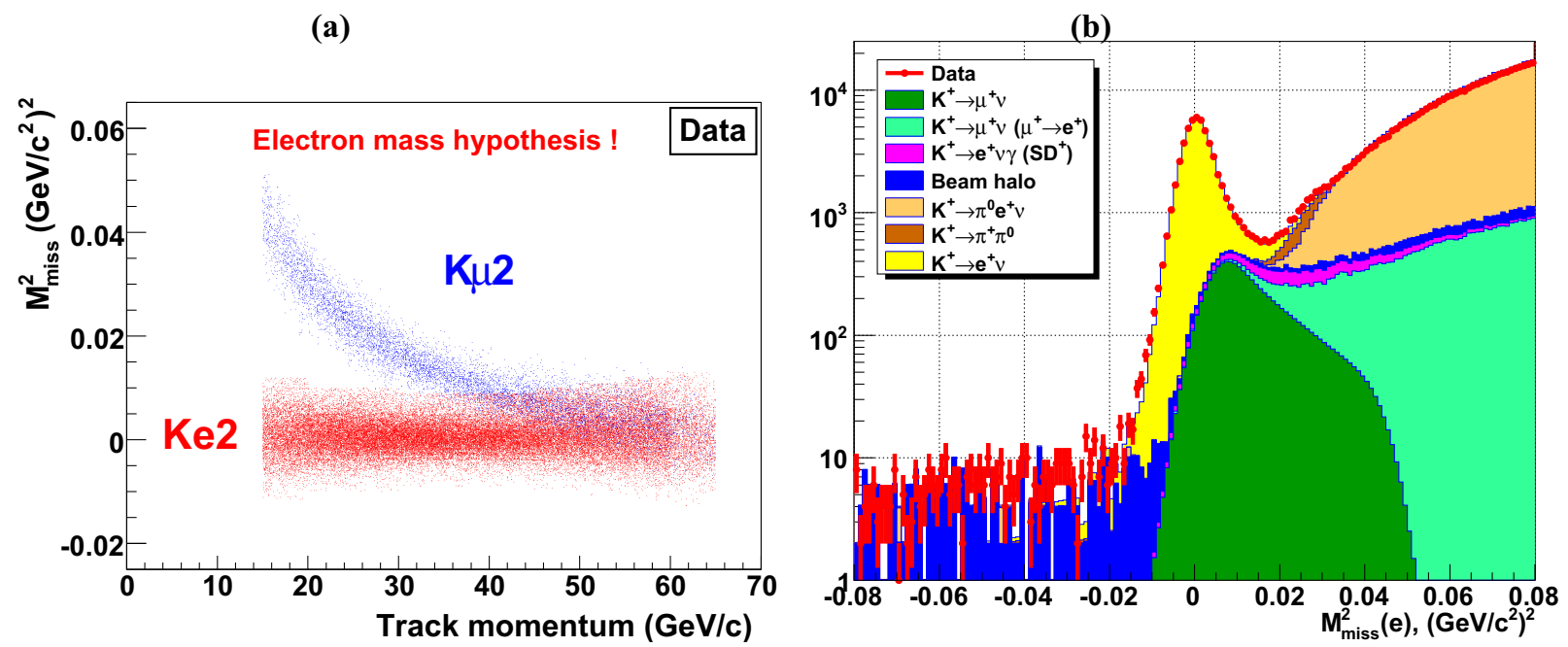

FIGURE 2. (a) Missing mass distribution for $K_{e 2}$ and $K_{\mu 2}$ simulated events as a function of the track momentum under electron hypothesis for the charged track. (b) Invariant mass distribution for the reconstructed data events (dots) compared with the expected signal and background contribution (histogram).

The experimentally measured ratio $R_{K}$ was determined as

$$
R_{K}=\frac{1}{D} \cdot \frac{N\left(K_{e 2}, \text { raw }\right)-N\left(K_{e 2}, \mathrm{bkg}\right)}{N\left(K_{\mu 2}, \text { raw }\right)-N\left(K_{\mu 2}, \mathrm{bkg}\right)} \cdot \frac{A\left(K_{\mu 2}\right) \times \varepsilon_{\text {trig }}\left(K_{\mu 2}\right) \times f_{\mu}}{A\left(K_{e 2}\right) \times \varepsilon_{\text {trig }}\left(K_{e 2}\right) \times f_{e}} \cdot \frac{1}{f_{l k r}},
$$

where $N\left(K_{\ell 2}\right.$, raw $), \ell=e, \mu$ is the number of the selected $K_{e 2}$ and $K_{\mu 2}$ candidates, $N\left(K_{\ell 2}, \mathrm{bkg}\right)$ is the number of expected background events, $f_{\ell}$ is the efficiency for particle identification, $A\left(K_{\ell 2}\right)$ is the geometrical efficiency for registration obtained from Monte Carlo simulation , $\varepsilon_{\text {trig }}$ is the trigger efficiency, $D=150$ is the downscaling factor for $K_{\mu 2}$ events and $f_{l k r}$ is the global efficiency of the LKR readout. All these quantities were obtained in bins of the charged track momentum. Based on $40 \%$ of the collected data the preliminary value for the $R_{K}$ is $R_{K}=\left(2.500 \pm 0.012_{\text {stat }} \pm 0.011_{\text {syst }}\right) \times 10^{-5}$ compatible with the SM predictions. Combining this value with the previous measurements the new world average for $R_{K}$ is $(2.498 \pm 0.014) \times 10^{-5}$. By using the whole data sample collected by NA62 in 2007 it will be possible to diminish the uncertainty on the ratio $R_{K}$ down to $0.4 \%$. 


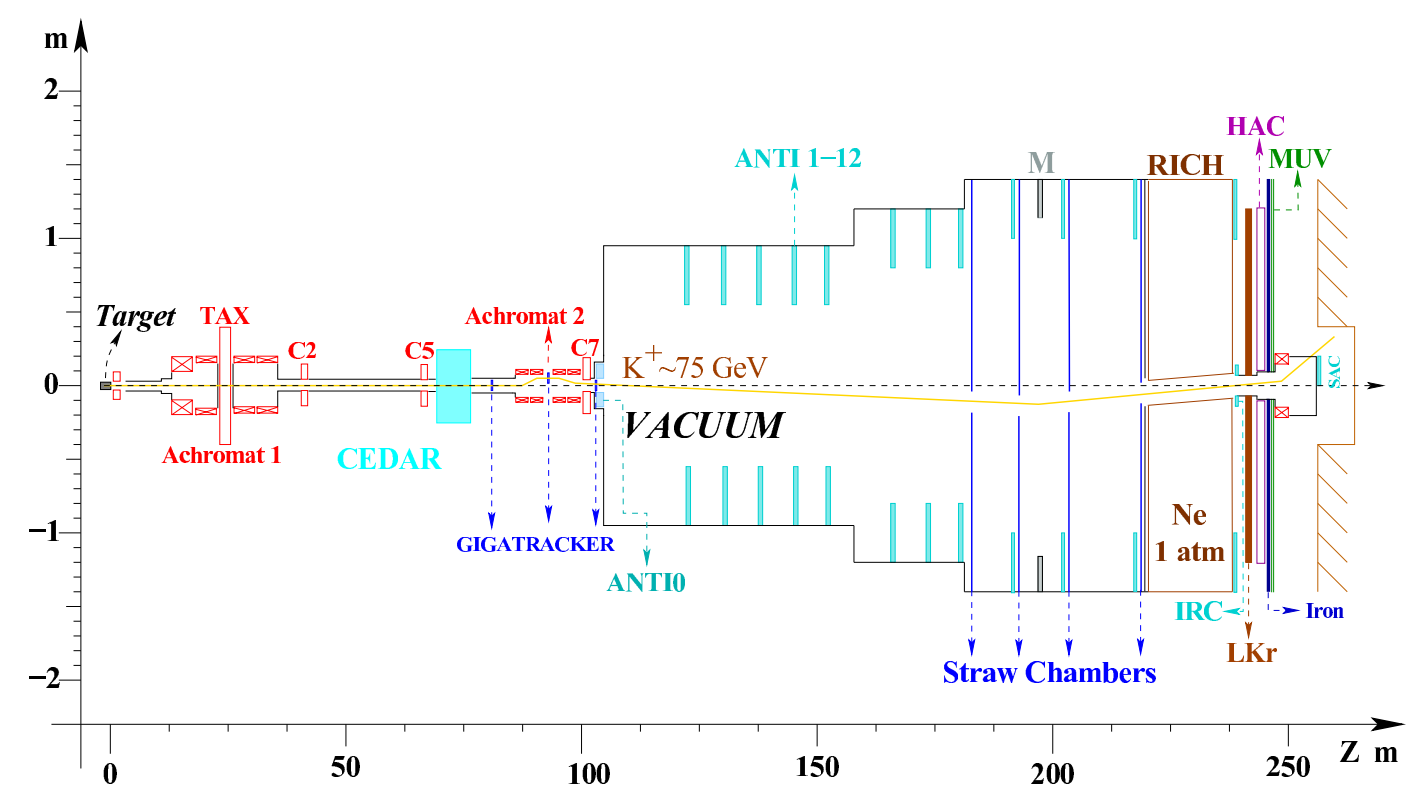

FIGURE 3. NA62 experimental layout

\section{THE DECAY $K^{ \pm} \rightarrow \pi^{ \pm} v \bar{v}$}

Among the rare kaon decays the transitions $K \rightarrow \pi v \bar{v}$ are extremely attractive. Their branching fractions are theoretically very clean since the hadronic matrix element can be obtained by the isospin symmetry of the strong interactions from the leading decay $K^{+} \rightarrow \pi^{0} e^{+} v$ [12]. For the charged kaon mode the NNLO calculations give $\operatorname{Br}\left(K^{ \pm} \rightarrow \pi^{ \pm} v \bar{v}\right)=(8.51 \pm 0.73) * 10^{-11}[13]$.

Presently seven $K^{ \pm} \rightarrow \pi^{ \pm} v \bar{v}$ events have been observed by the E787 and E949 collaborations in a stopped kaon experiment [14] leading to $\operatorname{Br}\left(K^{ \pm} \rightarrow \pi^{ \pm} v \bar{v}\right)=\left(1.73_{-1.05}^{+1.15}\right) \times 10^{-10}$. This value is twice the SM prediction but still compatible with it due to high uncertainty. The decay $K^{ \pm} \rightarrow \pi^{ \pm} v \bar{v}$ is very sensitive to New Physics models where the theoretical predictions vary over an order of magnitude. Thus measuring $\operatorname{Br}\left(K^{+} \rightarrow \pi^{+} v \bar{v}\right)$ could help to distinguish between the different types of New Physics [15] once it is discovered at the collider experiments.

\section{NA62 experiment}

In the final state there is only one observable particle. The kinematics variable considered for the separation of the decay is the missing mass squared under pion hypothesis for the charged track defined as

$$
m_{m i s s}^{2} \simeq m_{K}^{2}\left(1-\frac{\left|P_{\pi}^{2}\right|}{\left|P_{K}^{2}\right|}\right)+m_{\pi}^{2}\left(1-\frac{\left|P_{K}^{2}\right|}{\left|P_{\pi}^{2}\right|}\right)-\left|P_{K}\right|\left|P_{\pi}\right| \theta_{\pi K}^{2},
$$

where $P_{\pi}, P_{K}$ and $\theta_{\pi K}$ are the pion and kaon three momentum respectively and the angle between them. The signal region is defined by the edges of the kinematically limited in the $m_{\text {miss }}^{2}$ distribution kaon decays $K^{+} \rightarrow \mu^{+} v$, $K^{+} \rightarrow \pi^{+} \pi^{0}$ and $K^{+} \rightarrow \pi^{+} \pi^{+} \pi^{-}$. In order to suppress the background particle identification and a system of veto detectors will be used. The detector should be made hermetic for photons originating from $\pi^{0}$ in the decay region. The overall photon veto system has to provide an inefficiency less than $10^{-8}$ for a $\pi^{0}$ coming from $K^{+} \rightarrow \pi^{+} \pi^{0}$ decay. Decays with muons in the final state (like $K^{+} \rightarrow \mu^{+} v, K^{+} \rightarrow \pi^{+} \pi^{-} \mu^{+} v$ ) will be suppressed using a muon veto system for which the total inefficiency should be less less than $5 \times 10^{-6}$.

The proposed experimental setup [16] will be placed in the NA48 experimental hall and is shown on Figure 3. The positively charged kaons produced by the $400 \mathrm{GeV} / \mathrm{c}$ primary SPS proton beam on a beryllium target form about $6 \%$ of the secondary beam. They will be identified by the differential Cerenkov counter (CEDAR). A spectrometer placed at the second achromat and consisting of three Si pixel stations (Gigatracker) will be used for kaon momentum 
measurement. The sensor and the readout chip thickness will be $200 \mu \mathrm{m}$ and $100 \mu \mathrm{m}$ correspondingly in order to minimize the multiple scattering. Afterwards the kaons will undergo decay in vacuum. The momentum of the charged pion will be measured by a magnetic spectrometer composed by four straw tubes chambers with a hole in the centre, also placed in vacuum. Each chamber will include four views rotated at 45 degrees. The NA48 magnet will be used. The chambers will be displaced in such a way that the central holes follow the undecayed beam deflected in the magnetic field.

The photon veto system consists of twelve rings of large angle vetoes (ANTIs) surrounding the decay volume and the magnetic spectrometer, made of lead-glass blocks coming from the dismounted OPAL calorimeter; the LKR, which will play the role of a veto for photons originating from $K^{ \pm} \rightarrow \pi^{ \pm} \pi^{0}$ decays flying at angles between 1 and $15 \mathrm{mrad}$ with respect to the kaon flight direction and two small angle veto calorimeters (IRC and SAC) covering photon angles down to zero degree.

Particle identification will be accomplished by the usage of ring imaging Cerenkov counter (RICH) and the hadron calorimeter (HAC) and muon veto (MUV). The RICH is designed to provide $\pi^{+}-\mu^{+}$separation better than $1 \%$ and time resolution of 100ps. This will allow its usage also in the trigger. It will be filled with $\mathrm{Ne}$ at atmospheric pressure. HAC and MUV will profit from the existing NA48 hadronic calorimeter with two additional planes of fast scintillating detectors, placed after an iron wall. The inefficiency for muon detection of such device predicted by Monte Carlo simulation is about $10^{-5}$.

With the presented experimental setup and analysis technique the acceptance for the signal is about $14 \%$ with signal to background ratio of $\sim 10: 1$. The experiment was approved by the CERN Research Board, the construction will start in 2010 and data taking will be in 2012-2013. NA62 experiment will collect $\mathrm{O}(100) K^{ \pm} \rightarrow \pi^{ \pm} v \bar{v}$ events in two years of data taking assuming Standard Model branching fraction.

\section{CONCLUSIONS}

Kaon decays continue to provide valuable information to the physics of weak and strong interactions. Rare decays are unique possibility to study effects which could achieve sensitive contribution from New Physics processes and the high precision experiments are complementary to the high energy frontier. Once the New Physics is discovered at LHC the flavour physics will play a major role to disentangle among the different possible scenarios.

\section{ACKNOWLEDGMENTS}

The author thanks the organizers of the BPU7 conference. The presented work was partially supported by the Bulgarian Ministry of Science and Education under contract VUF-04/05 and by the University of Sofia under contract 260/15.05.2009.

\section{REFERENCES}

1. J.R.Batley et al., CERN/SPSC 2000-003 (2000).

2. V. Fanti et al. , Nucl. Instrum. Meth. A 574 (2007) 433.

3. CERN Program Library Long Write-up W5013 (1993).

4. G. DâĂŹAmbrosio et al., JHEP 9808 (1998) 4.

5. S. Friot et al. PLB 595 (2004) 301.

6. $\quad$ Dubnickova et al., Phys. Part. Nucl. Lett. 5 (2008) 76.

7. P. Bloch, et al., Phys. Lett. B 56 (1975) 201; C. Alliegro, et al., Phys. Rev. Lett. 68 (1992) 278; R. Appel, et al., Phys. Rev. Lett. 83 (1999) 4482.

8. M. Finkemeier, Phys. Lett. B 387 (1996) 391

9. V. Cirigliano and I. Rosell, Phys. Rev. Lett. 99 (2007) 231801.

10. A. Masiero et al., Phys. Rev. D 74 (2006) 011701

11. L. Fiorini, PoS HEP2005 (2006) 288 ; V. Kozhuharov, PoS (KAON) 049, arXiv:0710.5867 [hep-ex].

12. F. Mescia and C. Smith, Phys. Rev. D 76 (2007) 034017

13. M. Antonelli et al., arXiv:0907.5386 [hep-ph].

14. A. V. Artamonov et al., Phys. Rev. D 79 (2009) 092004

15. A. J. Buras et al, Rev. Mod. Phys. 80 (2008) 965

16. NA62 Collaboration, CERN-SPSC-2007-035, SPSC-M-760; 